\title{
Unsupervised image segmentation using a simple MRF model with a new implementation scheme
}

\author{
Huawu Deng*, David A. Clausi \\ Department of Systems Design Engineering, University of Waterloo, 200 University Avenue West, Waterloo, Ont., Canada N2L $3 G 1$
}

Received 15 October 2003; accepted 27 April 2004

\begin{abstract}
A simple Markov random field model with a new implementation scheme is proposed for unsupervised image segmentation based on image features. The traditional two-component MRF model for segmentation requires training data to estimate necessary model parameters and is thus unsuitable for unsupervised segmentation. The new implementation scheme solves this problem by introducing a function-based weighting parameter between the two components. Using this method, the simple MRF model is able to automatically estimate model parameters and produce accurate unsupervised segmentation results. Experiments demonstrate that the proposed algorithm is able to segment various types of images (gray scale, color, texture) and achieves an improvement over the traditional method.
\end{abstract}

(C) 2004 Pattern Recognition Society. Published by Elsevier Ltd. All rights reserved.

Keywords: Image segmentation; Unsupervised segmentation; Markov random field (MRF); Feature space; Expectation-maximization (EM) algorithm; K-means clustering; Synthetic aperture radar (SAR); Sea ice; Color image; Texture image

\section{Introduction}

Segmentation is a fundamental process in digital image processing which has found extensive applications in areas such as content-based image retrieval, medical image processing, and remote sensing image processing. Its purpose is to extract labelled regions or boundaries for targeted objects for subsequent processing such as surface description and object recognition.

A segmentation procedure usually consists of two steps. The first step is to choose a proper set of features which can identify the same-content regions and meanwhile differentiate different-content regions; the second step is to apply

\footnotetext{
* Corresponding author. Tel.: +1-519-888-4567-6377; fax: +1519-746-4791.

E-mail address: h2deng@engmail.uwaterloo.ca (H. Deng)
}

a segmentation method to the chosen features to achieve a segmentation map. Imagery in different applications varies and may require different methods to extract distinct features. Feature extraction is a broad topic and this paper will focus only on how to develop segmentation methods assuming the features used are sufficient to identify same-content regions and differentiate different-content regions.

Numerous segmentation methods have been proposed in the research literature, e.g., thresholding methods [1,2], clustering methods [3,4], edge-based methods [5], region splitting and merging methods [6-8], and multi-resolution techniques [9-11]. This paper addresses a Markov random field (MRF) based segmentation approach for segmenting synthetic aperture radar (SAR) sea ice imagery, color images and images with textures without supervision.

MRF is considered as a powerful stochastic tool to model the joint probability distribution of the image pixels in terms of local spatial interactions [12-14]. MRF models can be used not only to extract texture 
features from image textures but also to model the image segmentation problem, as from the viewpoint of the random field a segmentation result is a label distribution in the same lattice as the original image. Using MRF models for image segmentation has a number of advantages. First, the spatial relationship can be seamlessly integrated into a segmentation procedure. Second, the MRF based segmentation model can be inferred in the Bayesian framework which is able to utilize various kinds of image features. Third, the label distribution can be obtained when maximizing the probability of the MRF model [14].

There are various MRF based segmentation models that have been developed. Cohen and Cooper [15] proposed a doubly MRF model for segmenting range images and natural scenes. The doubly stochastic representation uses a Gaussian MRF to model textures and an auto-binary MRF to model a priori information about the local geometry of textured image regions. Won and Derin [16] developed a hierarchical MRF model for segmenting noisy and textured images. The model assumes the texture process also as a Gaussian MRF and can be used to segment images with GMRF-modelled textures very well. Geman et al. [17] constructed a joint MRF model according to a constrained optimization criterion. Panjwani and Healey [18] extended the coupled MRF models to segment textured color images. The MRF based segmentation model Barker [19] used is dynamic according to a model selection criterion implemented by a reversible jump method. Melas and Wilson [20] applied the double MRF model with modification to segment satellite images. A common point of the above applications is that the segmentation is highly dependent on the representability of the MRF parameters estimated from textures. Due to the variety of textures and non-stationarity property in most textures, the above MRF based segmentation models cannot work well for segmenting these images in which the textures cannot be modelled by MRF models. A practical MRF based model should be able to use different kinds of image features for different segmentation tasks [21].

This work formulates a simple MRF model which can easily utilize different kinds of image features. This MRF model consists of two components: a region labelling component and a feature modelling component. The region labelling component imposes a homogeneity constraint on the image segmentation process, while the feature modelling component functions as fitting the feature data. A constant weighting parameter is generally used to combine the two components. This model works very well if training data is available to estimate the parameters of both components. Practical applications are however increasingly required to work under unsupervised environment. That is to say, no training data is available and the segmentation procedure should have the ability to learn its parameters without human intervention. Under such unsupervised environment, the above model is not able to work consistently.

The root cause comes from how the two components interact to each other. Both components are probability dis- tributions and the interaction between them is represented by the product of two probability distributions. Each component therefore functions as a constraint for the other. A weighting parameter (as the power of the probability or the weight to its energy function) must be assigned to combine the two components in order to determine how much each component contributes to the whole system. With a constant weighting parameter, segmentation results can fall into three cases. If the constant parameter makes the region labelling component dominant, the values of parameters estimated may deviate too much from the real feature data. If the constant parameter makes the feature modelling component dominant, the spatial relationship information is ignored in the final segmented result. If a balance can be achieved between both components by choosing a proper constant parameter, the estimated parameters are not globally but locally optimal. All of these cases may generate inaccurate segmentation results.

This paper explores a new implementation scheme to combine the two components by introducing a variable weighting parameter between them. The variable parameter will first function as learning approximately globally optimal parameters. A balance is then achieved between the two components such that the spatial relationship information can be taken into consideration to refine the parameters when using a simulated annealing scheme for optimization. This approach is demonstrated to eventually generate more accurate segmentation results than the model with a constant parameter.

The rest of the paper is organized as follows. Section 2 discusses inference of a simple MRF based segmentation model for feature space and Section 3 discusses how to implement the segmentation model. Section 4 presents experiments of applying the segmentation model with the new implementation scheme to segment SAR sea ice imagery, color images, and images with textures. Conclusions are drawn in Section 5.

\section{Segmentation model}

\subsection{MRF basics}

In order to make this paper self-contained, a number of important concepts for MRF models are restated here. Readers are referred to [12-14] for details.

Let $S=\{s=(i, j) \mid 1 \leqslant i \leqslant H, 1 \leqslant j \leqslant W, i, j, H, W \in I\}$ be the set of image lattice sites, where $H$ and $W$ are the image height and width in pixels. In the two-dimensional image lattice $S$, the pixel values $x=\left\{x_{S} \mid s \in S\right\}$ are a realization of random variables $X=\left\{X_{S} \mid s \in S\right\}$.

Definition 1. A neighborhood system $N=\left\{N_{S}, s \in S\right\}$ is a collection of subsets of $S$ for which $s \notin N_{S}$ and $r \in N_{S} \Leftrightarrow$ $s \in N_{r} . N_{s}$ are the neighbors of $s$. 


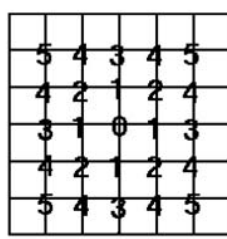

(a)

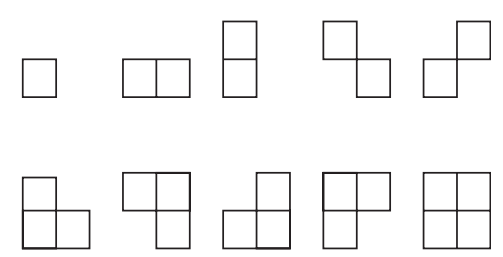

(b)
Fig. 1. (a) The fifth-order neighborhood system. (b) All cliques for the second-order neighborhood system.

Definition 2 (modified [22]). A $n$-th order neighborhood system is $N_{s}^{n}=\left\{s+\left.r\left|s+r \in N_{s},\right| r\right|^{2} \leqslant F[n]\right\}$, where $|r|$ denotes the Euclidian distance between sites $s$ and $s+r, F[n]$ is a member of the set of all possible integers defined as $F=\left\{F[n] \mid F[n]=i^{2}+j^{2}, i, j \in\right.$ $I, i+j>0, F[k]>F[l]$ if $k>l>0\}$. 1(a).

The fifth order neighborhood system is displayed in Fig.

Definition 3. A clique $c$ is a subset of $S$ for which every pair of sites are neighbors.

Definition 4. A clique set $C$ in the neighborhood system $N_{S}$ is $C=\left\{c \mid c \subset N_{S}\right\}$.

All possible cliques for the second-order neighborhood system is displayed in Fig. 1(b).

Definition 5. A random field $X$ is a Markov random field $(M R F)$ with respect to the neighborhood system $N=\left\{N_{S}, s \in\right.$ $S\}$ iff

1. $P(X=x)>0$ for all $x \in \Omega_{X}$, where $\Omega_{X}$ is the set of all possible $x$ on $S$;

2. $P\left(X_{s}=x_{s} \mid X_{r}=x_{r}, r \neq s\right)=P\left(X_{s}=x_{s} \mid X_{r}=x_{r}\right.$, $\left.r \in N_{S}\right)$.

Definition 6. $X$ is a Gibbs random field $(G R F)$ with respect to the neighborhood system $N=\left\{N_{S}, s \in S\right\}$ iff

$P(X=x)=\frac{1}{Z} \exp \left[-\frac{1}{T} U(x)\right]$

where $Z=\sum_{x \in \Omega} \exp [-(1 / T) U(x)]$ is a normalization constant, $T$ is the temperature parameter. $U(x)$ is the energy function with the form $U(x)=\sum_{c \in S} V_{c(x)}$, where $V_{c}(x)$ is a potential function.

Theorem 1 (Hammersley-Clifford). A random field $X$ is a GRF with respect to the neighborhood system $N=\left\{N_{s}, s \in S\right\}$ iff $X$ is an MRF with respect to $N=\left\{N_{s}, s \in S\right\}$.
The proof of Theorem 1 can be found in [13]. The theorem allows one to use a GRF which describes global characteristics of an image to represent the corresponding MRF that describes local characteristics of the image.

\subsection{A simple MRF based segmentation model}

The segmentation problem can be expressed in the Bayesian framework. Denote the feature vector extracted from a random image $(X=x)$ by $F=f$, where $F$ denotes a random variable and $f$ is an instance of $F . Y=y$ stands for a segmented result based on the feature vector $F=f$.

According to the Bayes rule, the segmentation problem is formulated as

$P(Y=y \mid F=f)=\frac{p(F=f \mid Y=y) P(Y=y)}{p(F=f)}$,

where $P(Y=y \mid F=f)$ is the posteriori probability of $Y=y$ conditioned on $F=f, p(F=f \mid Y=y)$ denotes the probability distribution of $F=f$ conditioned on $Y=y, P(Y=y)$ is a priori probability of $Y=y$, and $p(F=f)$ is the probability distribution of $F=f$.

A couple of assumptions should be made here to derive a simple MRF based segmentation model. The first assumption is that each component of $F=f$ be independent on the other components with respect to $Y=y$ (conditional independence). Suppose there are $K$ components in the feature vector $f=\left\{f^{k} \mid k=1,2, \ldots, K\right\}$. Eq. (2) is then transformed into:

$P(Y=y \mid F=f)=\frac{\prod_{k=1}^{K}\left[p\left(f^{k} \mid Y=y\right)\right] P(Y=y)}{p(F=f)}$,

where $p\left(f^{k} \mid Y=y\right)$ stands for the probability distribution of the extracted feature component $f^{k}$ conditioned on the segmented result $Y=y$.

As $F=f$ is known, $p(F=f)$ does not vary with respect to any solution $Y=y$ and hence can be disregarded since only the relative probability is of concern when maximizing $P(Y=y \mid F=f) . P(Y=y)$ describes the label distribution of a segmented result only and is normally referred to as the region labelling component. Most MRF based segmentation models use the MLL (multi-level logistic) model for modelling the label distribution. Generally, for a segmentation task, the second order pairwise MLL model is chosen and the potentials of all non-pairwise cliques are defined to be zeros [14]. The energy of the pairwise MLL model is as follows.

$E_{R}(y)=\sum_{s}\left[\beta \sum_{t \in N_{s}} \delta\left(y_{s}, y_{t}\right)\right]$,

where $\delta\left(y_{s}, y_{t}\right)=-1$ if $y_{s}=y_{t}, \delta\left(y_{s}, y_{t}\right)=1$ if $y_{s} \neq$ $y_{t}$, and $\beta$ is a constant which can be specified a priori [13]. $E_{R}(y)$ denotes the energy regarding image regions. By choosing this pairwise MLL model as the region labelling 
component in Eq. (2), the GRF form of $P(Y=y)$ is then expressed:

$P(Y=y)=\frac{1}{Z_{R}} \exp \left[-\frac{1}{T} E_{R}(y)\right]$,

where $Z_{R}=\sum_{y \in \Omega_{Y}}\left(1 / Z_{R}\right) \exp \left[-(1 / T) E_{R}(y)\right]$ is a normalization constant. $\Omega_{Y}$ stands for the set of all possible $Y=y$ on $S$.

Now only $p\left(f^{k} \mid Y=y\right)$ is unknown. Although most feature extraction methods are designed to extract a uniform response for all pixels in the class, the image features extracted often vary due to the existence of noise in the image and/or a non-stationary distribution of pixels in the samecontent region (Fig. 4). The feature data for one class can generally be assumed to be a normal distribution. Even if the distribution of a feature data is not a Gaussian distribution, the Gaussian function can still be used to approximate it since a unimodular distribution is expected. Here, the second assumption in this paper is to assume the distribution of all feature data be a Gaussian function with different means $\mu_{m}^{k}$ and standard deviations $\sigma_{m}^{k}$. That is,

$p\left(f_{s}^{k} \mid Y_{s}=m\right)=\frac{1}{\sqrt{2 \pi \sigma_{m}^{k}}} \exp \left[-\frac{\left(f_{s}^{k}-\mu_{m}^{k}\right)^{2}}{2 \sigma_{m}^{k^{2}}}\right]$,

where $\mu_{m}^{k}$ and $\sigma_{m}^{k}$ are the mean and standard deviation for the $m$ th class in the $k$ th feature component.

The product of all $p\left(f_{s}^{k} \mid Y_{s}=m\right)$ therefore describes the features for an image and are often referred to as the feature modelling component in Eq. (2). An energy form of this product is:

$E_{F}=\sum_{s, m=Y_{s}}\left\{\sum_{k=1}^{K}\left[\frac{\left(f_{s}^{k}-\mu_{m}^{k}\right)^{2}}{2\left(\sigma_{m}^{k}\right)^{2}}+\log \left(\sqrt{2 \pi} \sigma_{m}^{k}\right)\right]\right\}$.

The energy of $P(Y=y \mid F=f)$ is then derived

$E=E_{R}+\alpha E_{F}$,

where $\alpha$ is a weighting parameter to determine how much $E_{R}$ and $E_{F}$ individually contribute to the entire energy $E$. Its Gibbs form is $P(Y=y \mid F=f)=(1 / Z) \exp [-(1 / T) E]$, where $Z=\sum \Omega_{Y} \exp [-(1 / T) E]$. As there are two assumptions to simplify the feature data, the model in Eq. (8) is a simple MRF model.

\section{Implementation scheme}

\subsection{Maximum a posteriori criterion and simulated annealing}

One of the most important criteria for implementing MRF models is the maximum a posteriori (MAP) criterion [13].
For the model (8), the MAP may be any of the following:

$$
\begin{aligned}
\hat{y} & =\arg \max _{y \in \Omega_{Y}} P(Y=y \mid F=f) \\
& =\arg \max _{y \in \Omega_{Y}} \frac{1}{Z} \exp \left[-\frac{1}{T} E\right] \\
& =\arg \min _{y \in \Omega_{Y}} E .
\end{aligned}
$$

Eq. (9) means that maximizing the posteriori conditional probability distribution or Gibbs distribution is equivalent to minimizing the energy of the model.

If the energy function is convex, it is easy to achieve the global minimum of the energy by deterministic algorithms (such as ICM [12]). In most cases, however, the energy function is non-convex, hence the above algorithms may settle at a local minimum [23]. Two sampling methods are generally used to implement the MAP criterion: the Gibbs sampler [13] and the Metropolis sampler [24]. With an annealing scheme used for the Gibbs/Metropolis sampler, the convergence to the global minimum (energy) is guaranteed $[13,25]$.

When an annealing scheme is used in the Gibbs sampler/Metropolis sampler, its convergence holds under two conditions: infinite time or iterations for the Gibbs/Metropolis sampler and a decreasing temperature schedule which meets the condition $T(t) \geqslant N \Delta / \log (t+1)$, for $t \geqslant 1$, where $N$ is the image size and $\Delta$ is a norm of the difference between the maximum and minimum energy $[13,25]$. Readers are referred to [23] and [25] for the theorem about the necessary and sufficient conditions for the annealing scheme. Unfortunately, those theoretical schemes are of little practical use since (a) $\Delta$ is impossible to calculate in practice and (b) too many iterations are required to descend over a sufficient range of temperatures $[23,25]$.

Scientists have attempted to find fast annealing schemes. The most commonly used fast simulated annealing (SA) scheme is the logarithmic scheme proposed by Geman and Geman [13],

$T(t)=\frac{C}{\log (t+1)}, \quad C$ is a constant, and $t \geqslant 1$.

Numerous applications have demonstrated that using this logarithmic scheme can reach a suboptimal result within limited iterations $[13,25]$. This paper employs this logarithmic scheme to optimize the simple MRF model and generate segmentation results.

\subsection{Parameter estimation}

Four parameters should be estimated: $\beta$ (from Eq. (4)), $\alpha$ (from Eq. (8)), $\mu, \sigma$. Estimation of $\mu$ and $\sigma$ for each class requires training data. However, under unsupervised environment, training data is not available. The expectation-maximization (EM) algorithm [26,27] is used to estimate $\mu$ and $\sigma$ and is able to obtain a segmentation map. The EM algorithm for the simple MRF model 
(Eq. (8)) is outlined as follows:

1. A random segmentation image is initialized.

2. E-step: Estimate $\mu$ and $\sigma$ from the feature data $F=f$ based on the segmented image:

$\mu_{m}^{k}=\frac{1}{N} \sum_{s, Y_{s}=m} f_{s}^{k}, \sigma_{m}^{k}=\left[\frac{1}{N-1} \sum_{s, Y_{s}=m}\left(f_{s}^{k}-\mu_{m}^{k}\right)^{2}\right]^{\frac{1}{2}}$.

3. M-step: Use the estimated $\mu$ and $\sigma$ to obtain a refined segmentation image by minimizing Eq. (8) using the Metropolis sampling with a simulated annealing scheme.

4. Repeat the above two steps until a stopping criterion is satisfied.

The difficulty is that there is no closed-form solution for $\beta$ and $\alpha$ in the EM algorithm. A commonly used strategy [13] is to priorily assign values to them by experience before executing the EM algorithm. Both parameters $\beta$ and $\alpha$ function in the same manner by assigning weights to their corresponding energy components, and hence one of them can be fixed. Here, $\beta$ is fixed to be 1 and only $\alpha$ is required to be adjusted. As the weighting parameter $\alpha$ is normally set as a constant parameter, the segmentation result often falls into three cases.

First, if the constant parameter makes the region labelling component dominant, the values of estimated parameters $\mu$ and $\sigma$ may deviate much from the feature data and the segmented result is not consistent. Fig. 3 demonstrates outcomes using the MLL model alone (which is equal to setting $\alpha=0$ ) to generate segmented results. Two tests are shown: (1) starting from the same random image but at different iterations and (2) starting from different random images but at the same number of iterations. These tests demonstrate that the segmented results are quite different for the same segmentation task due to the lack of feature data.

Second, if the constant parameter makes the feature modelling component dominant, spatial relationship information would be ignored in the final segmented result. For example, if setting $\beta=0$ and $\alpha \neq 0$, the MRF model has the feature modelling component only and is unable to produce a segmented result. Third, if a balance can be achieved between both components by choosing a proper constant parameter, the estimated parameters are normally not globally but locally optimal. An example for segmenting a checkerboard image can demonstrate that. Fig. 4(a) is a three-class checkerboard image corrupted by an additive noise. Three modes can be clearly found in the histogram (Fig. 4(b)) of this noisy checkerboard image. With a random initial label image and choosing a proper constant for the parameter $\alpha$ $(\alpha=8)$, the result shown in Fig. 4(c) is incorrect in that the estimated parameters (means) of two classes are confused.

The root cause is that the simple MRF based segmentation model is very easily trapped in local maxima due to the imposed spatial homogeneity constraint by the region labelling component. As a result, the feature modelling component might not be able to learn the global parameters (i.e. $\mu$ and $\sigma$ for each class). Stewart et al. [28] analyzed the relationship between the two terms in their MRF model in detail and proposed a specific solution for the weighting parameter (they called it shape parameter) according to a priori information of the size of region shapes.

A new implementation scheme is proposed here to solve this problem by making the weighting parameter $\alpha$ vary during unsupervised segmentation. The introduction of the variable weighting parameter can not only enable the segmentation procedure to learn the global parameters of the feature modelling component but also impose spatial homogeneity constraint on the label distribution (through the region labelling component). For such purpose, the parameter should vary with respect to the annealing procedure. This work chooses the following function for the variable weighting parameter $\alpha$ :

$\alpha(t)=c_{1} 0.9^{t}+c_{2}$,

where $c_{1}$ and $c_{2}$ are constants. In this experiment, $c_{1}=80$ and $c_{2}=1$. The later experiments also demonstrate $c_{1}=80$ is appropriate for most cases and $c_{2}=1 / K$ where $K$ is the number features used for segmentation. With the above function, the feature modelling component will first (when $\alpha(t)$ is larger) dominate the simple MRF model in order to learn its global parameters and then (when $\alpha(t)$ is close to $c_{2}$ ) interacts with the region labelling component to refine the segmented result.

Thus the energy of the simple MRF model can be rewritten as:

$E=E_{R}+\alpha(t) E_{F}$

By using the simple MRF model with the variable weighting parameter, Fig. 4(a) can be well segmented. The result displayed in Fig. 4(d) demonstrates the mean and standard deviation of each class are properly estimated and the segmented regions are uniform respectively. This is a dramatic improvement over the standard model displayed in Fig. 4(c).

\section{Experimental results}

\subsection{Testing methodology}

Three methods are used for image segmentation: (1) the simple MRF model with a variable weighting parameter, (2) the simple MRF model with a constant weighting parameter, and (3) the K-means clustering method [29].

The first two segmentation methods are implemented by the EM algorithm: an iteration of E- and M-step as discussed earlier (in Section 3). The constant $C$ (i.e., the initial temperature) of the logarithmic annealing scheme is set to 2 in the M-step following Geman and Geman's experiments [13]. A fixed number of iterations is used as the stopping criterion in 


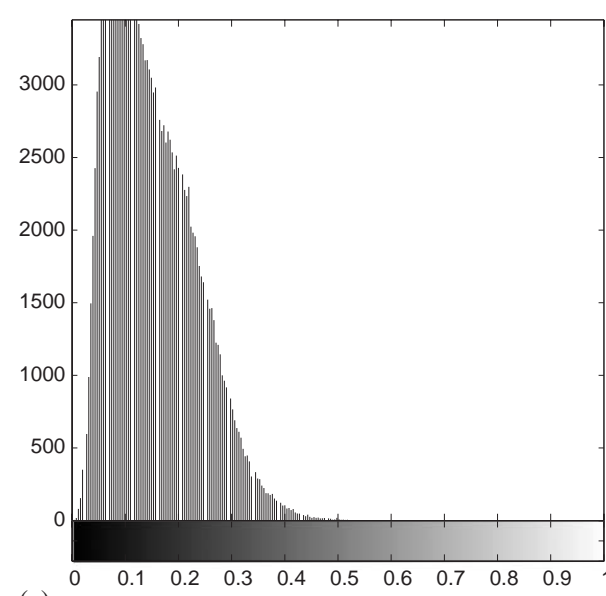

(a)

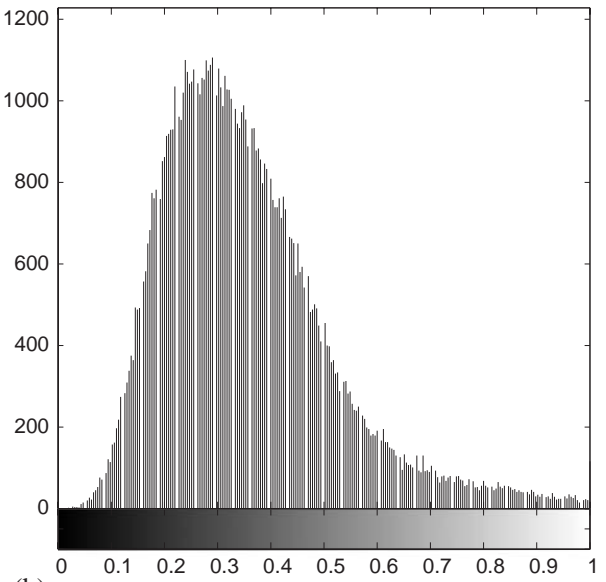

(b)

Fig. 2. (a) Histogram of the image in Fig. 5. (b) Histogram of the image in Fig. 9 (up-left).

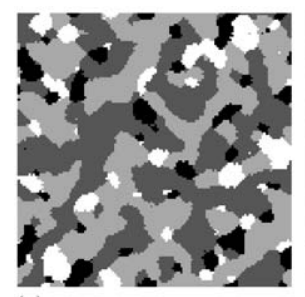

(a)

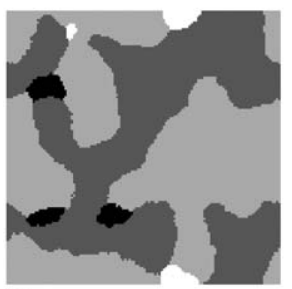

(e)

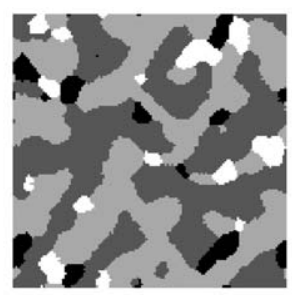

(b)

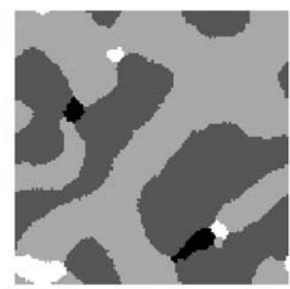

(f)

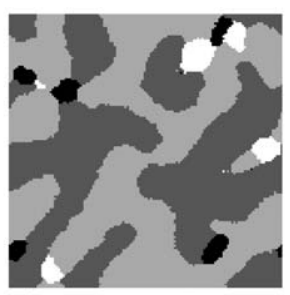

(c)

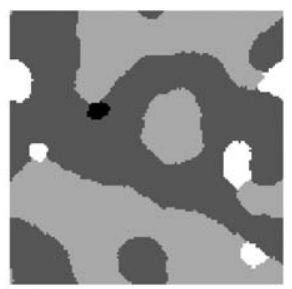

(g)

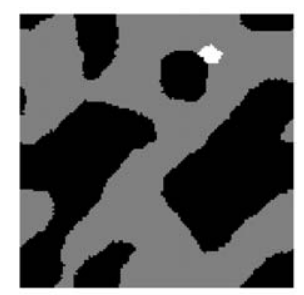

(d)

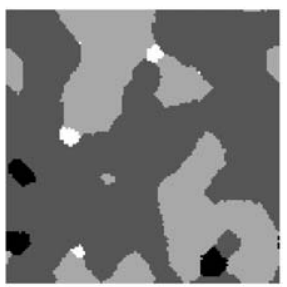

(h)

Fig. 3. First Row-Segmented results from the same random initial at different iterations: (a) 50 iterations; (b) 100 iterations; (c) 200 iterations; (d) 400 iterations. Second Row (e)-(h): Segmented results from different random initials at the 300th iteration, respectively.

the following experiments. The tests conducted in this work indicate that segmented results will change appreciably after 150 iterations and the result at the 150th iteration can be considered as final. The K-means clustering method is also iterative. Its stopping criterion is that the clustering procedure stops when there is no change for the label of every pixel. The initial seed for the clustering is chosen randomly.

Three types of images are tested: synthetic aperture radar (SAR) sea ice images, color images, and images with textures. All three segmentation methods are provided the number of classes depending on the specific image. The final segmented results will be presented in a segmented map (for segmentation of images with textures) or boundaries superimposed over the original image (for SAR sea ice imagery and color image segmentation).

\subsection{SAR sea ice imagery segmentation}

Operationally, numerous SAR images are interpreted daily in support of sea ice monitoring. One important application of the SAR sea ice imagery is to measure, monitor, and understand of sea ice evolution during the seasons and a fundamental step of this application requires generation of ice type maps. In the future, computer assisted segmentation techniques are expected to contribute to this task.

There are two types of sea ice images considered in this work for segmentation. One kind of sea ice images consist of ice and water regions. The task for segmenting the ice-water images is to separate ice regions from water regions to determine the ice concentration. Another type of sea ice image 


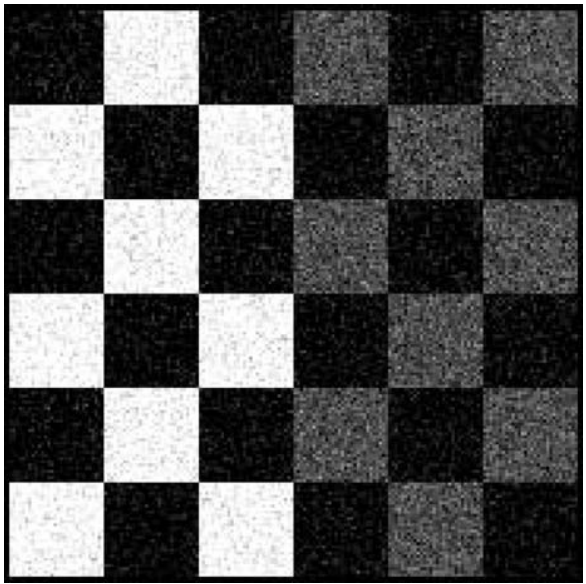

(a)

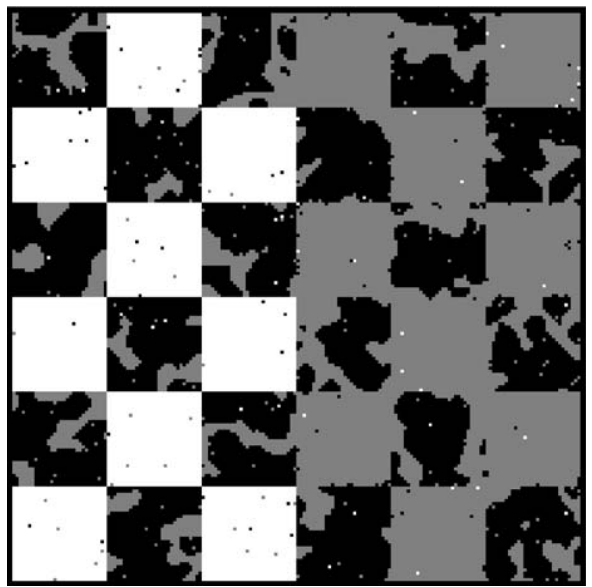

(c)

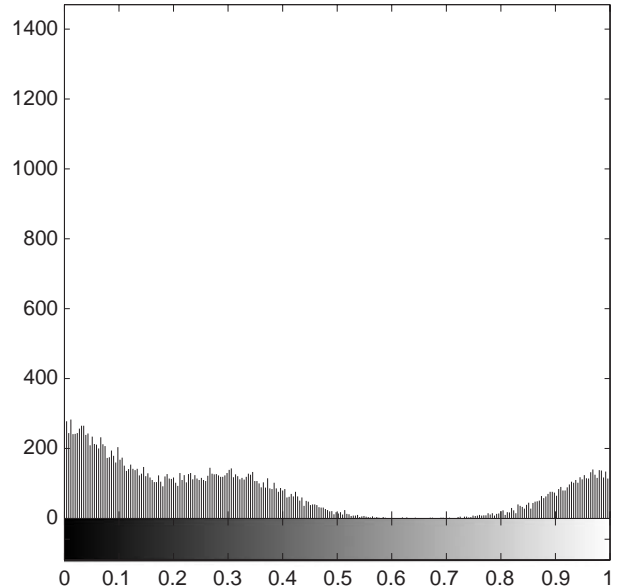

(b)

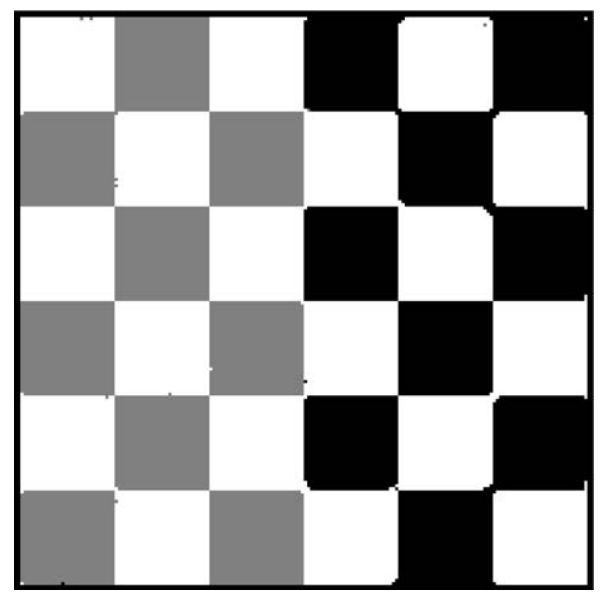

(d)

Fig. 4. (a) Original three-class checkerboard image corrupted by an additive noise; (b) histogram; (c) segmented result by the simple MRF model with a constant weighting parameter $(\alpha=8)$; (d) segmented result by the simple MRF model with a variable weighting parameter $\left(\alpha(t)=80 \times 0.9^{t}+1\right)$.

consists of different types of sea ice and is required in practice to extract boundaries between sea ice types and partition the corresponding regions. The proposed segmentation approach is applied to segment these sea ice images when using image intensity only as image feature. Texture features should be used for more complicated SAR sea ice images, but there is insufficient space to implement those in this paper.

Two images extracted from two SAR sea ice scenes respectively are tested. The first image shown in Fig. 5 is part of the scene of a C-band HH Radarsat ScanSAR data (100 m pixel spacing) covering Baffin Bay and Davis Strait captured on June 24, 1998. Its histogram of pixel intensity is shown in Fig. 2(a). The segmentation task for the first image is to extract ice regions (light gray areas) from open water regions (dark gray areas). Fig. 6 shows the result seg- mented by the simple MRF based segmentation model with the variable weighting parameter. Fig. 7 shows the best result among these obtained by the simple MRF model using different constant weighting parameters. It can be seen that the segmented result has many small regions. This is because the constant weighting parameter makes the region labelling component contribute less energy to the whole system than the feature modelling component so that the final segmented result does not incorporate sufficient spatial relationship information. Fig. 8 shows the result obtained by the K-means clustering method. As the K-means clustering method does not take into consideration of spatial relationship at all, the segmented result has more small regions. Since most of these small regions are generally caused by noise, they should be removed in practice. By visually comparing Fig. 6 with Figs. 7 and 8, it is obvious that using 


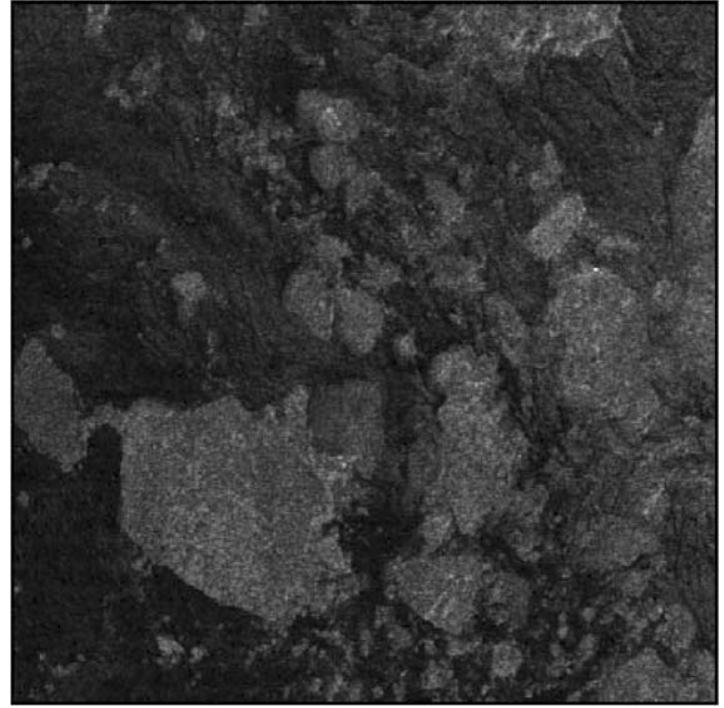

Fig. 5. Original SAR sea ice image with ice regions (light gray areas) and open water regions (dark gray areas).

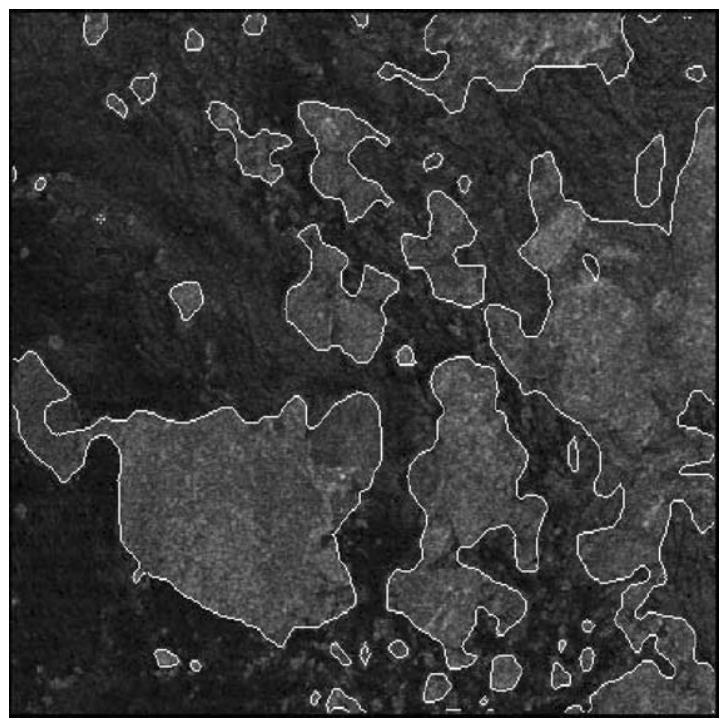

Fig. 6. Segmented result by the simple MRF model with a variable weighting parameter $\left(\alpha(t)=80 \times 0.9^{t}+1\right)$.

the simple MRF model with the variable weighting parameter generates much more accurate result than the other two algorithms.

The second image shown in Fig. 9 (up-left) is part of the scene of a C-band HH Radarsat ScanSAR data (100 m pixel spacing) covering Baffin Bay and Davis Strait acquired on February 7, 1998. This image consists of three types of sea ice (multi-year ice (white areas), rough first-year ice (light gray areas), and smooth first-year ice (dark gray areas)) which are required to be partitioned from each other.

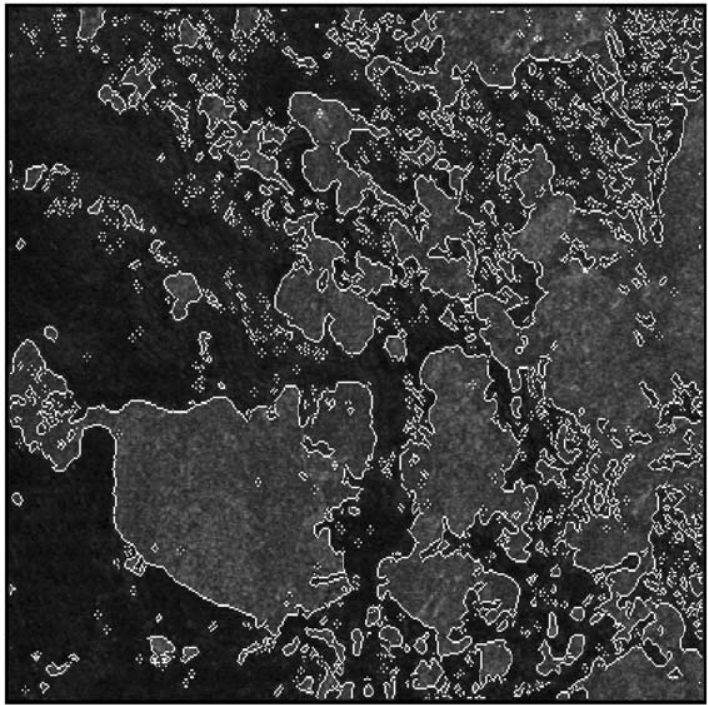

Fig. 7. Segmented result by the simple MRF model with a constant weighting parameter $(\alpha=6)$.

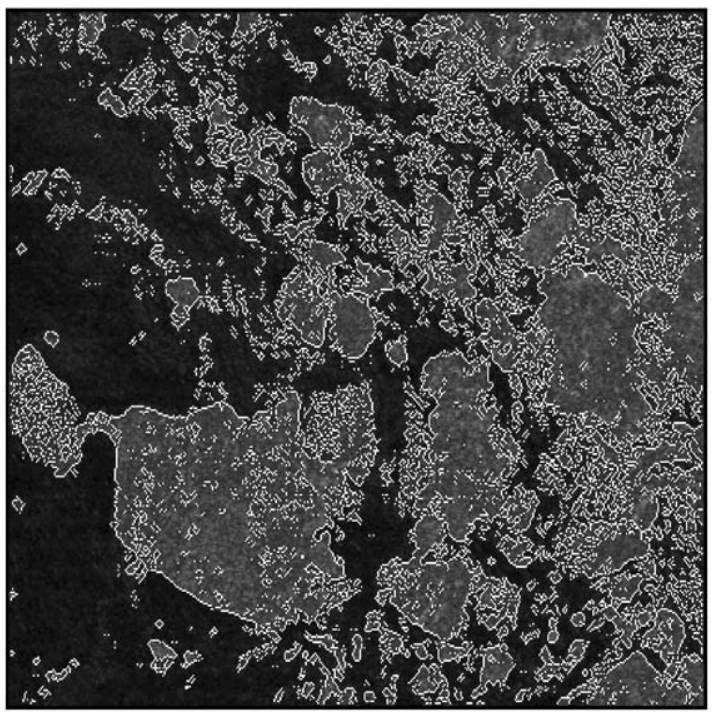

Fig. 8. Segmented result by the K-means clustering method.

Fig. 2(b) plots its histogram of pixel intensity. The above three methods are applied and the segmented results are shown in Fig. 9 (up-right, bottom-left, bottom-right). As there is large intensity variance in these pixels which actually belongs to the same ice type, the spatial homogeneity constraint on neighboring pixels are very important for clustering the same-class pixels. Therefore, by comparing the three results, the K-means clustering method generates the most inaccurate result where the means of three ice types are confused. The result by the simple MRF model 

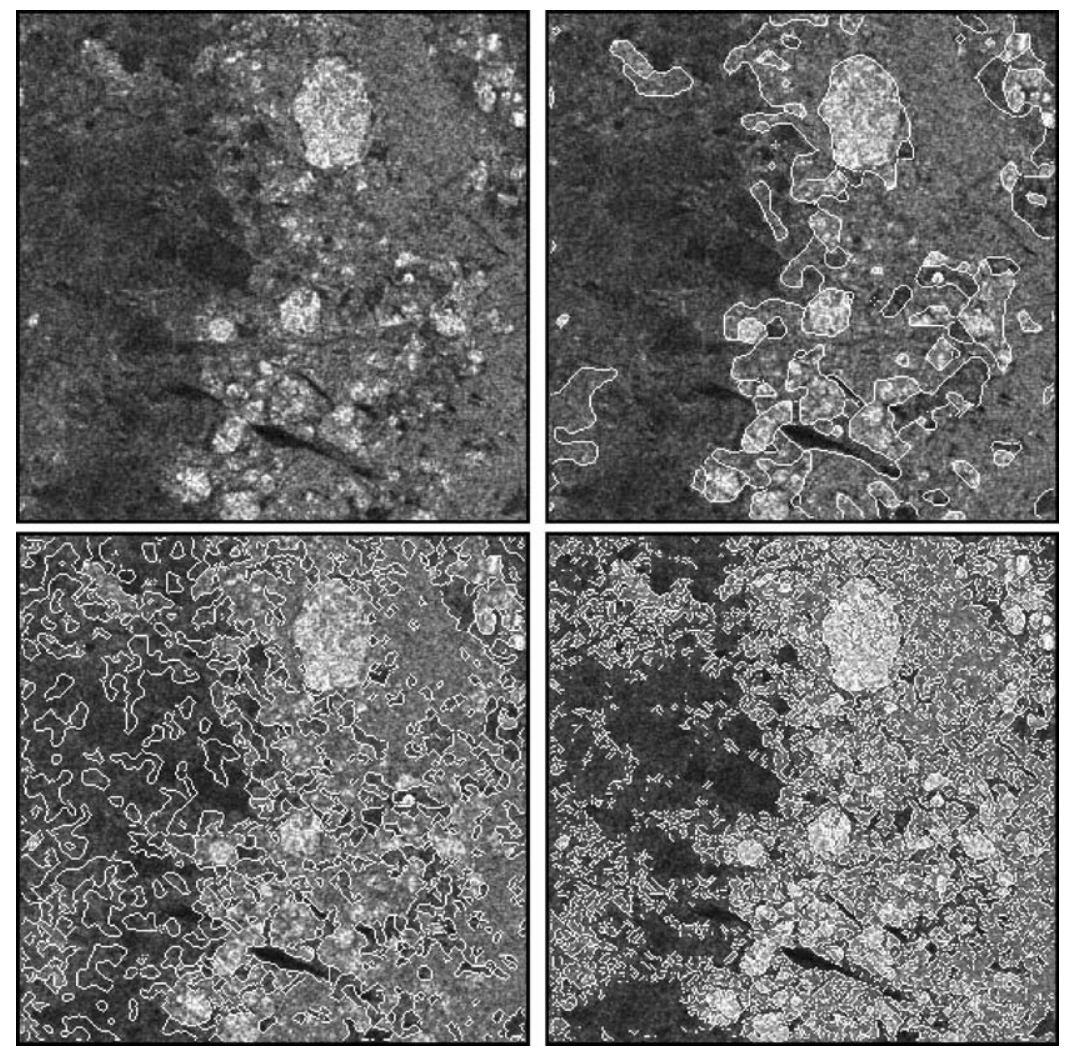

Fig. 9. Up-left: Original SAR sea ice image with three types of sea ice (multi-year ice (white areas), rough first-year ice (light gray areas), and smooth first-year ice (dark gray areas)). Up-right: Segmented result by the simple MRF model with a variable weighting parameter $\left(\alpha(t)=80 \times 0.9^{t}+1\right)$. Bottom-left: Segmented result by the simple MRF model with a constant weighting parameter $(\alpha=8)$. Bottom-right: Segmented result by the K-means clustering method.

with a constant weighting parameter has some improvement over that by the K-means clustering method, but the means of three ice types are still confused. Using the variable weighting parameter, the simple MRF model can generate the most accurate result in that the three ice types are clearly identified.

\subsection{Color image segmentation}

As an important cue for visual discrimination ability for human being, color can be used as features for segmenting natural color images. The segmented map can then be used to extract object shape or refine extracted features in terms of object classification. For example, this procedure is an initial and significant step in content-based image retrieval systems.

There are a number of color spaces to quantize color information, e.g., RGB (Red/Green/Blue), HSV (Hue/Satuation/Value of intensity), L*a*b (Lightness/greenred/blue-yellow), CMYK (pure Cyan/Magenta/Yellow/ blacK) and so on. RGB and HSV are two commonly used color spaces. However, colors in the HSV space are more appropriate to be used as features for classification/segmentation purpose because each component in the RGB color space often has high correlation with the others and the HSV color space shows very consistency to human vision systems [30].

In these experiments, each color image is converted to the HSV color space and the HSV values will be used as color features to segment the image. Four images of natural scenes are tested and shown here. The boundaries between different regions in the segmented result are superimposed on the original image for a clear view. The simple MRF model with a variable weighting parameter is applied. For comparison, the simple MRF model with a constant weighting parameter is used to segment the four images. In Fig. 10, the images in the left column are the four original images, and the images in the middle column are the results segmented by the simple MRF model with the variable weighting parameter $\left(\alpha(t)=80 \times 0.9^{t}+\frac{1}{3}\right)$, and the images in the right column are the segmented results by the simple MRF model with a constant weighting parameter $(\alpha=2)$. 

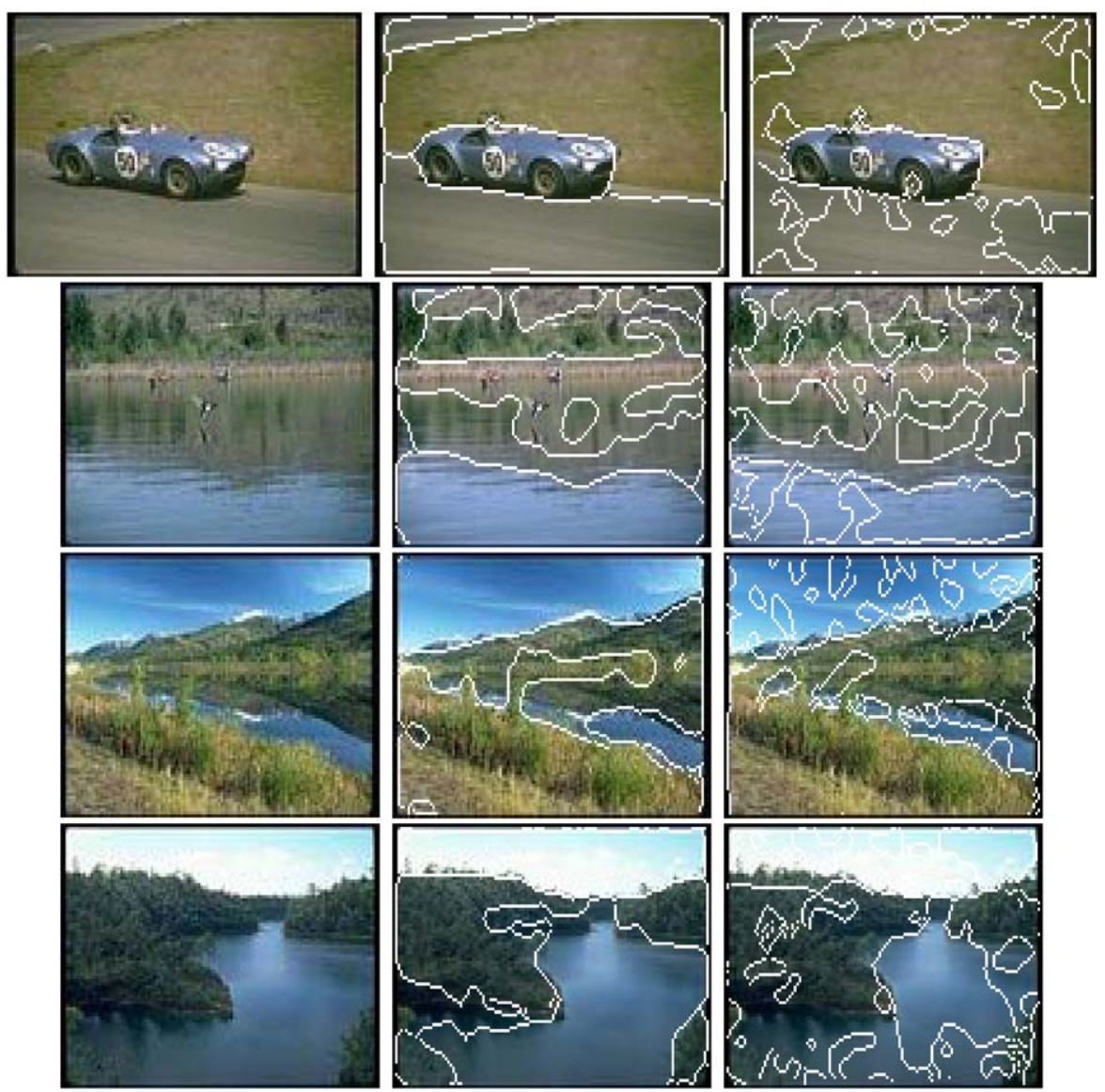

Fig. 10. Left column: Original color images; middle column: segmented results by the simple MRF model with a variable weighting parameter $\left(\alpha(t)=80 \times 0.9^{t}+1 / 3\right)$; right column: segmented results by the simple MRF model with a constant weighting parameter $(\alpha=2)$.

The first image has three objects to be partitioned: car, road and grass. The simple MRF model with the constant weighting parameter cannot separate the grass from the road but generates new classes in the road and in the grass (shown in the right-hand-side image). This is caused by the large variation of intensities in the same-class pixels. By using the variable weighting parameter, the simple MRF model can successfully differentiate the grass and land (shown in the middle image).

The second image is assigned to obtain four classes: water with inverted sky, water with inverted trees, trees, and mountainside. Due to being unable to learn a global mean for each class, the simple MRF model with the constant weighting parameter partitions the single region into other small different regions (shown in the right-hand-side image). In contrast, the simple MRF model with the variable weighting parameter can identify the regions of water with inverted sky, trees, and mountainside and obtain most of the region of water with inverted trees (shown in the middle image).

The third image is required to obtain four regions: sky, light grass, dark mountain with grass, and lake with inverted sky. The use of the constant weighting parameter makes the simple MRF model partition the sky region into several small different regions (shown in the right-hand-side image), while the use of the variable weighting parameter enables the simple MRF model to generate a uniform sky region (shown in the middle image).

The fourth image has three classes to be separated: sky, trees on island, and water with inverted sky. The simple MRF model with the constant parameter fails to identify two of them (shown in the right-hand-side image), but the use of the variable parameter makes the simple MRF model segment all regions correctly (shown in the middle image).

\subsection{Segmentation of images with textures}

Segmentation of images with textures is often a very difficult task. Although the main difficulty is in finding proper features to represent textures, another problem about how to apply a segmentation method to the extracted feature space, which is normally high-dimensional, can affect the final segmentation performance directly. Here, the simple MRF 


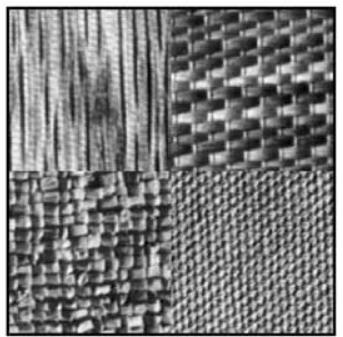

(a)

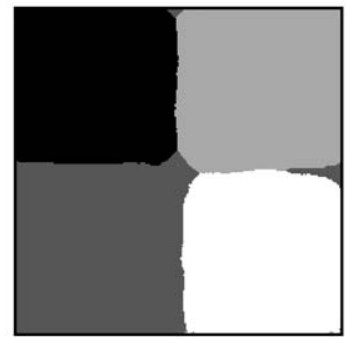

(b)

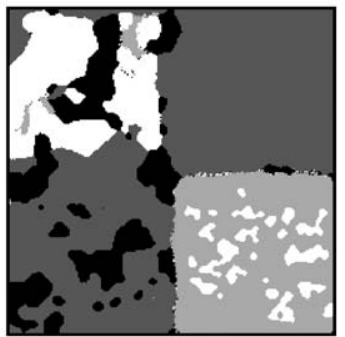

(c)

Fig. 11. (a) Original image with four textures in square regions; (b) segmented result by the simple MRF model with a variable weighting parameter $\left(\alpha(t)=80 \times 0.9^{t}+1 / 24\right)$ with $97 \%$ accuracy rate; (c) segmented result by the simple MRF model with a constant weighting parameter $(\alpha=1)$ with $65 \%$ accuracy rate.

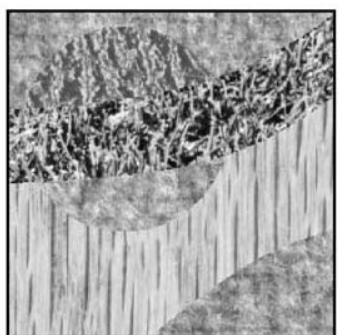

(a)

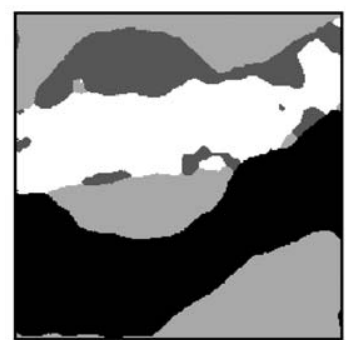

(b)

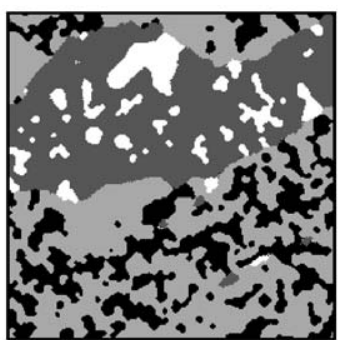

(c)

Fig. 12. (a) Original image with four textures in different boundary shapes; (b) segmented result by the simple MRF model with a variable weighting parameter $\left(\alpha(t)=80 \times 0.9^{t}+1 / 24\right)$ with $91 \%$ accuracy rate; (c) segmented result by the simple MRF model with a constant weighting parameter $(\alpha=1)$ with $58 \%$ accuracy rate.

model is used as a segmentation method to partition images with textures.

Research has demonstrated that the human visual system (HVS) is sensitive to both specific orientations and spatial frequencies. For texture analysis, wavelets have the ability to model the frequency and orientation sensitivity characteristic of the HVS [31]. The Gabor filter bank can be designed to mimic a wavelet filter bank. Due to its appealing simplicity and optimum joint spatial/spatial-frequency localization, the Gabor function is attractive for computer vision applications, especially texture segmentation $[32,33]$.

In this work, a total of 24 complex Gabor filters at four frequencies $(22.63,11.31,5.66,2.83 \mathrm{ppc}$ (pixels per cycle)) (corresponding to the frequencies $8 \sqrt{2}, 16 \sqrt{2}, 32 \sqrt{2}$, $64 \sqrt{2}$ cycles per image for a $256 \times 256$ image) and six orientations $\left(0^{\circ}, 30^{\circ}, 60^{\circ}, 90^{\circ}, 120^{\circ}, 150^{\circ}\right.$ are chosen to filter a test image [34]. The magnitude of each filtered image will be smoothed by an amplitude Gaussian (the scale is $\frac{2}{3}$ [33]). Thus a 24-D Gabor filter feature vector can be obtained for each pixel. The simple MRF model with the variable weighting parameter is applied to the 24-D Gabor filter feature space to obtain a segmentation map. For com- parison, the simple MRF model with the constant weighting parameter is also applied to segment the target images.

Two images with different textures are tested by both segmentation algorithms. Figs. 11 and 12 display the corresponding results. Fig. 11(a) is an image with four Brodatz textures [35]. This original image was used as a test image in Mao and Jain's paper [36]. It can be seen that the simple MRF model with the constant parameter is trapped in local minima and hence cannot identify the means of three classes (shown in Fig. 11(c) with $65 \%$ accuracy rate only). Using the variable parameter however enables the simple MRF model to learn the appropriate global mean for each class and leads to an accurate segmentation result (shown in Fig. 11(b) with 97\% accuracy rate).

The second image (shown in Fig. 12(a)) was used in the paper [37] which provides a variety of textures and boundary shapes. Four classes of texture regions are required to be clustered. The simple MRF model with the constant weighting parameter fails to differentiate the four classes (shown in Fig. 12(c) with 58\% accuracy rate only), while using the variable weighting parameter makes the simple MRF model successfully identify most of the four classes (shown in Fig. 12(b) with $91 \%$ accuracy rate). 


\section{Conclusions}

By introducing a variable weighting parameter to combine the region labelling component and the feature modelling component in a simple MRF based segmentation model, an unsupervised segmentation can be achieved. Experiments demonstrated that the new implementation scheme can enable the simple MRF model to work more consistently than a constant weighting parameter. The developed technique can be efficiently applied to SAR sea ice imagery segmentation, color image segmentation and segmentation of images with textures.

\section{Acknowledgements}

The authors are thankful to GEOIDE (http://www.geoide. ulaval.ca/) and CRYSYS (http://www.crysys.ca) for their financial support and CIS (http://www.cis.ec.gc.ca/) for providing the SAR sea ice images (CCSA http://www.space.gc.ca).

\section{References}

[1] P.K. Sahoo, S. Soltani, A. Wong, Y. Chen, A survey of thresholding techniques, Comput. Vision, Graphics Image Process. 41 (1988) 233-260.

[2] J.S. Wezka, A survey of threshold selection techniques, Comput. Vision, Graphics Image Process. 7 (1978) 259-265.

[3] D.A. Clausi, K-means iterative fisher (KIF) unsupervised clustering algorithm applied to image texture segmentation, Pattern Recognition 35 (9) (2002) 1959-1972.

[4] A.K. Jain, R.P.W. Duin, J.C. Mao, Statistical pattern recognition: a review, IEEE Trans. Pattern Anal. Mach. Intell. 22 (1) (2000) 4-37.

[5] J. Basak, B. Chanda, D.D. Manjumder, On edge and line linking with connectionist models, IEEE Trans. Systems, Man Cybernet. 24 (3) (1994) 413-428.

[6] Y.L. Chang, X.B. Li, Adaptive image region-growing, IEEE Trans. Image Process. 3 (6) (1994) 868-872.

[7] S.A. Hojjatoleslami, J. Kittler, Region growing: a new approach, IEEE Trans. Image Process. 7 (7) (1998) $1079-1084$.

[8] X.L. Wu, Adaptive split-and-merge segmentation based on piecewise least-square approximation, IEEE Trans. Pattern Anal. Mach. Intell. 15 (8) (1993) 808-815.

[9] C.A. Bouman, B. Liu, Multiple resolution segmentation of textured images, IEEE Trans. Pattern Anal. Mach. Intell. 13 (2) (1991) 99-113.

[10] C.A. Bouman, M. Shapiro, A multiscale random field model for Bayesian image segmentation, IEEE Trans. Image Process. 3 (2) (1994) 162-177.

[11] M. Mignotte, C. Collet, P. Perez, P. Bouthemy, Sonar image segmentation using an unsupervised hierarchical MRF model, IEEE Trans. Image Process. 9 (7) (2000) 1216-1231.

[12] J.E. Besag, Spatial interaction and the statistical analysis of lattice systems (with discussion), J. R. Stat. Soc. B-36 (1974) 192-236.
[13] S. Geman, D. Geman, Stochastic relaxation, Gibbs distributions, and the Bayesian restoration of images, IEEE Trans. Pattern Anal. Mach. Intell. 6 (6) (1984) 721-741.

[14] S.Z. Li, Markov Random Field Modeling in Computer Vision, Springer, New York, 2001.

[15] F.S. Cohen, D.B. Cooper, Simple parallel hierarchical and relaxation algorithms for segmenting noncausal Markovian random fields, IEEE Trans. Pattern Anal. Mach. Intell. 9 (2) (1987) 195-219.

[16] C.S. Won, H. Derin, Unsupervised segmentation of noisy and textured images using Markov random fields, CVGIP: Graphical Models Image Process. 54 (4) (1992) 308-328.

[17] D. Geman, S. Geman, C. Graffigne, P. Dong, Boundary detection by constrained optimization, IEEE Trans. Pattern Anal. Mach. Intell. 12 (7) (1990) 609-628.

[18] D.K. Panjwani, G. Healey, Markov random field models for unsupervised segmentation of textured color images, IEEE Trans. Pattern Anal. Mach. Intell. 17 (10) (1995) 939-954.

[19] S.A. Barker, Image segmentation using Markov random field models, Ph.D. Thesis, University of Cambridge, 1998.

[20] D.E. Melas, S.P. Wilson, Double Markov random fields and Bayesian image segmentation, IEEE Trans. Signal Process. 50 (2) (2002) 357-365.

[21] P.P. Raghu, B. Yegnanarayana, Segmentation of Gabor-filtered textures using deterministic relaxation, IEEE Trans. Image Process. 5 (12) (1996) 1625-1635.

[22] H.W. Deng, D.A. Clausi, Advanced Gaussian MRF rotationinvariant texture features for classification of remote sensing imagery, in: Proceedings of IEEE Computer Society Conference on Computer Vision and Pattern Recognition (CVPR), Vol. II, June 2003, Madison, USA,2003, pp. 685-690.

[23] P.J.M. van Laarhoven, E.H.L. Aarts, Simulated Annealing Theory and Applications, D. Reidel Publishing Company, Dordrecht, Holland, 1987.

[24] N. Metropolis, A.W. Rosenbluth, M.N. Rosenbluth, A.H. Teller, E. Teller, Equations of state calculations by fast computing machines, J. Chem. Phys. 21 (1953) 1087-1091.

[25] G. Winkler, Image Analysis, Random Fields and Dynamic Monte Carlo Method, Springer, New York, USA, 1995.

[26] A.P. Dempster, N.M. Laird, D.B. Rubin, Maximum likelihood from incomplete data via the EM algorithm, J. R. Stat. Soc. B-1 (1977) 1-38.

[27] J. Zhang, The mean field theory in EM procedures for Markov random fields, IEEE Trans. Signal Process. 40 (10) (1992) 2570-2583.

[28] D. Stewart, D. Blacknell, A. Blake, R. Cook, C. Oliver, Optimal approach to SAR image segmentation and classification, IEE Proc.-Radar, Sonar Navigation 147 (3) (2000) 134-142.

[29] R.O. Duda, P.E. Hart, Pattern classification and scene analysis, A Wiley Iinterscience Publication, USA, 2000.

[30] J.M. Corridoni, A.D. Bimbo, P. Pala, Image retrieval by color semantics, Multimedia Systems 7 (2) (1999) 175-183.

[31] A.R. Rao, G.L. Lohse, Identifying high level features of texture perception, CVGIP: Graphical Models Image Process. 55 (3) (1993) 5218-5233.

[32] A.K. Jain, F. Farrokhnia, Unsupervised texture segmentation using Gabor filters, Pattern Recognition 24 (12) (1991) 1167-1186. 
[33] A.C. Bovik, M. Clark, W.S. Geisler, Multichannel texture analysis using localized spatial filters, IEEE Trans. Pattern Anal. Mach. Intell. 12 (1) (1990) 55-73.

[34] D.A. Clausi, M.E. Jernigan, Designing Gabor filters for optimal texture separability, Pattern Recognition 33 (11) (2000) 1835-1849.

[35] P. Brodatz, Texture-A Photographic Album for Artists and Designers, Reinhold, New York, USA, 1968.
[36] J. Mao, A.K. Jain, A self-organizing network for hyperellipsoidal clustering (HEC), IEEE Trans. Neural Networks 7 (1) (1996) 16-29.

[37] S. Krishnamachari, R. Chellappa, Multiresolution Gauss-Markov random field models for texture segmentation, IEEE Trans. Image Process. 6 (2) (1997) 251-267.

\begin{abstract}
About the Author-HUAWU DENG obtained his B.A.Sc. in 1992 from Beijing University, China, M.Eng. in 1995 from Research Institute of Petroleum Exploration and Development, China, and Ph.D. in 2001 from Nanyang Technological University, Singapore. From 2000 to 2002, he worked as an information technology analyst for a regional bank in Singapore. He is now with University of Waterloo, Canada, as a post-doctoral research fellow. His current research interests are in image processing (texture modelling, image classification, image segmentation, image retrieval), computer vision, pattern recognition, and remote sensing data analysis.
\end{abstract}

About the Author-DAVID A. CLAUSI earned his B.A.Sc. (1990), M.A.Sc. (1992), and Ph.D. (1996) in Systems Design Engineering at the University of Waterloo (Waterloo, Ont., Canada). After completing his doctorate, Prof. Clausi worked in the medical imaging field at Mitra Imaging Inc. (Waterloo). He started his academic career in 1997 as an Assistant Professor in Geomatics Engineering at the University of Calgary, Alberta, Canada. In 1999, he returned to his alma mater as an Assistant Professor in Systems Design Engineering and was awarded tenure and promotion to Associate Professor in 2003. Prof. Clausi is an active interdisciplinary and multidisciplinary researcher. He has a prestigious publication record, publishing refereed journal and conference papers in the diverse fields of remote sensing, computer vision, algorithm design, and biomechanics. His primary research interest is the automated interpretation of synthetic aperture radar (SAR) sea ice imagery, in support of operational activities of the Canadian Ice Service. The research results have led to successfully commercial implementations. He has received numerous graduate scholarships, awards for two conference papers, and a Teaching Excellence Award. 